\title{
Guidance of Clinical Magnet Application of Inhibition of Inappropriate Shock in Patient of Subcutaneous Implantable Cardioverter Defibrillators: A Review of the Literature
}

Santomauro Maurizio ${ }^{1 *}$, Cacciatore Francesco ${ }^{2}$, Riganti Carla ${ }^{3}$, Santomauro Andrea ${ }^{4}$, Castellano Gaetano ${ }^{5}$, Iovino Gianluigi ${ }^{1}$, Maffettone Francesco ${ }^{1}$, Piccolo Andrea ${ }^{1}$ and Abete Pasquale ${ }^{2}$

${ }^{1}$ Department of Cardiovascular Emergency, Internal Medicine and Geriatric, University of Naples, Italy

${ }^{2}$ Department of Translational Medical Sciences, Federico II University of Naples, Italy

${ }^{3}$ General Direction, Medical School, Federico II University of Naples, Italy

${ }^{4}$ Department of Medicine, Surgery, Dentistry, Salernitana Medical School, Italy

${ }^{5}$ Department of Anesthesia and Intensive Care, Gemelli Molise Hospital, Italy

*Corresponding author: Santomauro Maurizio, Department of Cardiovascular Emergency, Internal Medicine and Geriatric, Medical School, Federico II University of Naples, Italy.

Received Date: March 01, 2021

Published Date: March 22, 2021

\section{Abstract}

Background: The subcutaneous implantable cardioverter defibrillator (S-ICD) represents a major advancement in ICD technology in the last 10 years. During the experience with the $1 \mathrm{nd}$ generation of S-ICD, the IAS rate was 4,5\% to $25 \%$ and with 2 nd generation and 3nd generation of S-ICD the IAS rate was $3.1 \%$ at one year. Indeed, in the hospital and out of hospital emergency situations in presence of IAS it is mandatory to suspend the shock therapy.

Objective: In this study we performed a review of literature provides a comprensive overview of the current literature on the specific responses of S-ICD to clinical magnets to IAS reduce in the emergency situations.

Methods: A systematic search was limited between 2010 to 2020 in the database.

Results: Few prospective reports with a small number of patients have been published, revealing no relevant adverse events in patients with IAS. Six case reports on direct magnet application in S-ICD patients have been published, with many of them occurring in older devices and IAS.

Conclusion: In cases of cardiac and extracardiac malfunctions in S-ICD patients, a magnet can be used as a first step therapy to temporarily deactivate shocks in emergency situations. In the absence of a specific Boston Scientific magnet, any magnet can be used interchangeably for S-ICD deactivation therapy.

Keywords: Subcutaneous implantable cardioverter defibrillator; Inappropriate shock; Clinical magnet; Deactivation shock therapy

\section{Introduction}

The subcutaneous implantable cardioverter defibrillator (S-ICD) was approved for the prevention of sudden cardiac death among candidates to a transvenous implantable cardioverter defibrillator (T-ICD) without indication for anti-bradycardia pacing 
or cardiac resynchronisation therapy, recurrent monomorphic ventricular tachycardia (VT) responsive to antitachycardia pacing (ATP), or pre-existing unipolar pacemaker leads [1-5]. Clinical scenarios in an Emergency Department, such as cardiac and extracardiac oversensing that interfere with the device functioning or patients presenting with IAS, warrant reprogramming of S-ICD [6-13]. Device reprogramming is usually performed using the corresponding manufacturer's programmer, which is complex and requires technically trained personnel [14-16]. Alternatively, these devices are incorporated with magnet-sensitive switches that respond to clinical magnets to temporarily inhibit the shock therapy. Magnets are readily available and do not require special training to use, making them an excellent option to reprogram S-ICD devices in emergency situations. In general, the application of a magnet switches pacemakers to an asynchronous pacing mode and suspends all antitachycardia therapies of T-ICD [17-22]. In S-ICD instead, magnet application suspends the shock therapy. In this study we performed a systematic review provides a comprensive overview of the current literature on the specific responses of S-ICD to clinical magnets.

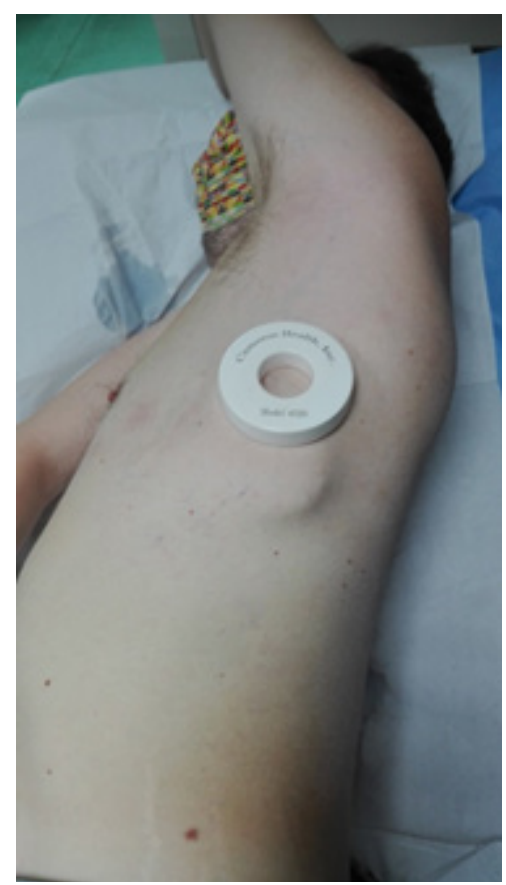

Figure 1A: Emblem S-ICD A209 - the magnet should be applied on the upper edge.

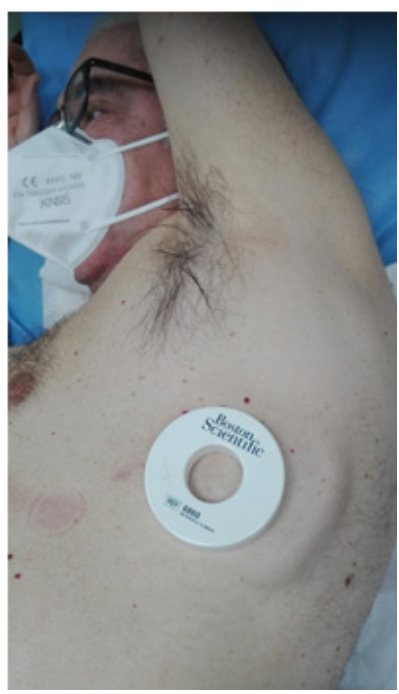

Figure 1B: Emblem S-ICD A219 - the magnet should be applied on the lower edge.

Figure 1: Correct position of specific Boston magnet. 


\section{Clinical Magnet for Suspension of Therapy: technical considerations}

All S-ICD have a built-in magnetic reed switch that is designed to switch 'ON' or 'OFF' circuitry in response to magnets. The Boston Scientific magnet 6860 Model and 4520 Model are accessories that may be used to temporarily inhibit the delivery of shock therapy from the device if necessary [23]. For the A209 EMBLEM S-ICD Model (2nd generation) and the A219 EMBLEM MRI S-ICD Model (3rd generation), it is necessary to apply the magnet flat on the skin over the device header (Figure 1A) or over the lower edge of the device (Figure 1B). The magnetic field effect of the clinical magnet is directly proportional to the strength of the magnet and inversely proportional to the distance between the magnet and the device. A magnetic field effect of $\geq 10$ Gauss aligned with the magnetic reed switch is required to activate the magnetic switch in order to alter the device function [23]. The site of magnet placement is important since a poorly positioned magnet may not produce the desired effect. If the magnet is correctly placed over the device, beeping tones (R-wave synchronous) will be heard approximately one second after the magnet is applied. Shock therapy is not suspended until beeping tones are heard. When using the magnet for a patient with a deep implant placement, the exact location of the device may not be evident and another magnet position may need to be tested near the general pulse device location. Beeping may be difficult to hear and a stethoscope should be used if necessary. If the beeper is disabled or if the patient has been through a magnetic resonance imaging (MRI) scan, the beeper may be inaudible [23,24]. It may be necessary to use the 3200 programmer (Boston Scientific) to suspend therapy in these patients (Figure 2). Beeping will continue for 60 seconds while the magnet is held in place. After 60 seconds, the beeping stops but therapy continues to be inhibited unless the magnet has been removed. If it is necessary to confirm whether the therapy is still being inhibited after the beeping has stopped, remove and reapply the magnet to reactivate the beeping tones. In patients with a deep implant placement, magnet application may fail to elicit the switch response. In this case, the magnet cannot be used to inhibit therapy. When long duration therapy suspension is desired, it is recommended to modify device behaviour with the 3200 programmer rather than the magnet. When the magnet is removed, arrhythmia detection resumes and therapy delivery is no longer inhibited. The effect of the magnet on the device varies depending on the mode the device is programmed. The Shelf mode puts the device on standby, reducing battery consumption and is exclusively intended for storage purposes. Programmer commanded rescue shocks and manual shocks are delivered if they are commanded with the magnet already in place. If the magnet is applied during a treated or untreated episode, the episode will not be stored in the device memory. Magnet application does not affect wireless communication between the device and the programmer. MRI Protection Mode modifies certain device functions in order to mitigate risks associated with exposing the S-ICD system to the MRI environment [24]. Choosing MRI Protection Mode will initiate a sequence of screens to assess the patient's eligibility and readiness to undergo an MR Conditional MRI scan. Prior to the patient undergoing an MRI scan, an ImageReady S-ICD System must be programmed to the MRI Protection Mode using the programmer. In MRI Protection Mode, tachycardia therapy is suspended, with programmable values of $6,9,12$, and 24 hours, the beeper is disabled. MRI Protection Mode is terminated by manual exit or through the user-programmed automatic MRI Protection Time-out period. Rescue Shock will also terminate during MRI Protection Mode. When MRI Protection Mode is exited, all parameters return to the previously programmed settings. The Beeper can be reenabled after exiting MRI Protection Mode.

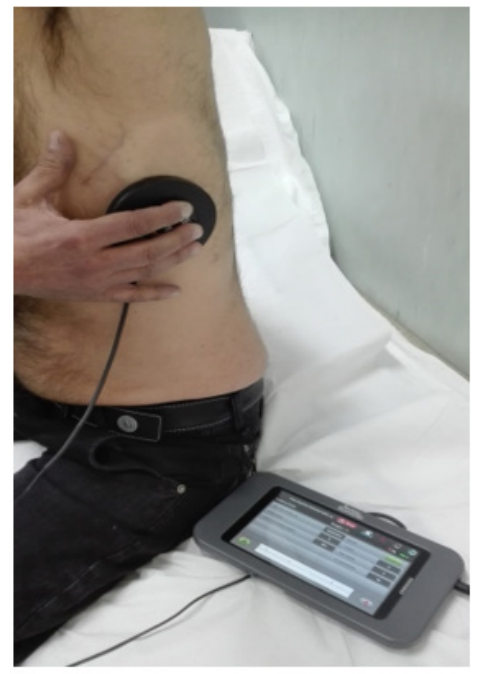

Figure 2: Correct telemetry position of 3200 programmer. 


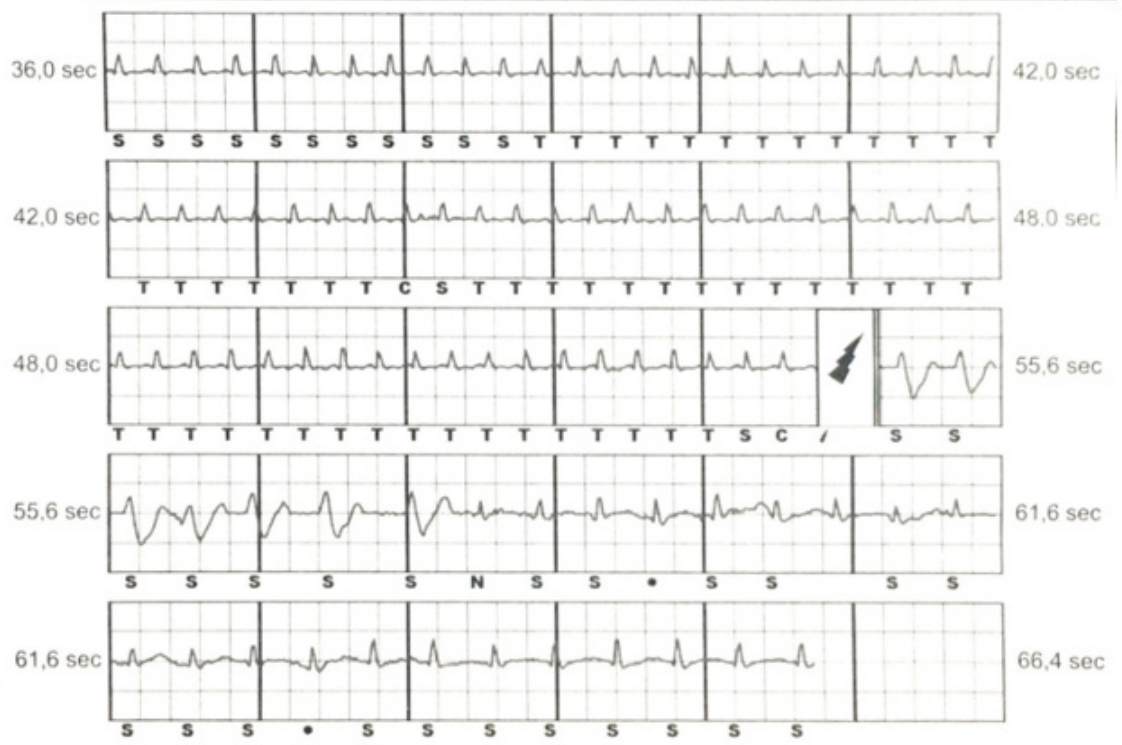

Figure 3: ECG stored the IAS in patient with atrial fibrillation episode.

\section{Method}

A systematic search was limited between January 1, 2010 to December 31, 2020 in the following database: PubMed, Embase. com (Elsevier), the Cochrane Library (Wiley), CRD (centre for Reviews and Dissemination): DARE (Database of Abstracts of Reviews of Effects, HTA (Health Technology Assessment Database). It was performed to identify articles with S-ICD and IAS rates by 3 investigators. The following Boolean search terms were utilized: "subcutaneous implanted cardioverter defibrillator", "inappropriate shock", "clinical magnet", "suspension of antitachy therapy" and " emergency electrophysiology procedures". By handsearch records identified through database searching resulting in 48 citations overall. Overall, 42 citations were identified after the removal of duplicates. The references were screened by two independent researchers (AS and FM) and, in case of disagreement, a third researcher (UA) was involved to resolve the differences. The selection process (PRISMA Flow Diagram) and methodology were approved by all authors. Titles and abstracts retrieved in the search were reviewed, and observational and comparative studies reporting IAS in S-ICD patients were selected. For IAS analysis case reports, review articles, abstracts, meta-analysis and editorials were included. In the event that there were multiple publications from the same study, the latest study with the most complete data available was selected, and the other publications were not used in order to avoid overlapping cohorts. For included studies, only data on IAS in S-ICD patients were extracted. Extracted data included: namely, IAS, S-ICD model, patients' mean age, arrhythmia detection, magnet application response. Data were extracted by one author and were reviewed by additional authors.

\section{Result}

After excluding 42 articles for not meeting inclusion/exclusion criteria, 6 case reports remained to be assessed for eligibility. Years of enrolment for the studies ranged from 2010 to 2020. Numerous case reports on direct magnet application in S-ICD patients exist, with many of them occurring in older devices and IAS [17,18, 27 30]. But, only a few prospective reports with a small number of patients have been published, revealing no relevant adverse events in patients with IAS [7-14].

\section{Discussion}

An S-ICD has two main hardware components: a defibrillator and a lead. The defibrillator is battery-powered and is surgically placed beneath the patient's skin. The lead is also implanted, with wires running from the defibrillator to the patient's extraheart. In patients when IAS are experienced, physicians have been directed to reprogram the device to decrease the likelihood of inappropriate activation (Figure 3). These shocks are painful, have the potential to induce lethal arrhythmias and can be extremely distressful psychologically to the patient [15]. Therefore, IAS causes myocardial injury (troponin release or ST-segment changes consistent with injury currents) and increases mortality $[25,26]$. During the experience with the 1nd generation of S-ICD, the IAS rate was high, with a risk of $4,5 \%$ to $25 \%$ [2-5]. A comprehensive review of the literature shows that $3.1 \%$ of patients with 2 nd generation and 3nd generation of S-ICD experienced their first IAS annually [6]. The recommended reprogramming is intended to reduce oversensing, which triggers multiple shocks [9]. It's extremely important to immediately place a patient suspected of having an S-ICD problem on a cardiac monitor. The best and most 
reliable sign that an S-ICD is malfunctioning is the absence of VT or ventricular fibrillation (VF) on cardiac monitor prior to the S-ICD firing. Physicians must always assume the device is functioning correctly unless there's proof from the cardiac monitor that the shock was inappropriately administered. If the S-ICD firing has been stopped by the emergency medical department (EMD) staff, two other pieces of information may help determine whether the firing was appropriate or not. Patients with S-ICD are advised to carry a card identifying their device, and they may be able to present this to EMD staff. Another piece of key information to obtain during assessment is whether any cardiac symptoms were present prior to the first shock. If the patient was not experiencing any cardiac signs and symptoms, the possibility of IAS should be considered. S-ICD are typically programmed during standard follow-up appointments. Appropriate placement of the magnet is variable depending on the device S-ICD model [18]. This variability in magnet placement increases the risk of failed device deactivation. For obese patients, to reduce the distance between the magnet and the device, we suggest in these cases, to manually modify the position of the device inside the muscular pocket, until the beeping sound is heard, with the aid of a stethoscope if necessary. Should the magnet position shift during a long procedure, there is no indication that the device has been reactivated and the patient is at risk for IAS. In preoperative setting, the S-ICD should be disabled by a programmer, external defibrillation therapy should be available at all times and the device should be reprogrammed immediately after surgery [27]. Due to limited practitioner medical experience with S-ICD and an inability to reprogram the device in emergency situations, a magnet was placed to prevent delivery of IAS. The lack of appreciation of the device's audible beeping, represents a potential mechanism for these unexpected shocks (Figure 4). Magnet deactivation of S-ICD is less reliable and should be used also during cardiopulmonary reanimation (CPR) manuvers [28,29]. Cmorej [30] reported a case of IAS in a patient with out-of-hospital sudden cardiac arrest, in whom shocks were induced during a verified asystole by chest compressions during CPR. During chest compression, the bystanders received a shock delivered by the S-ICD. In this experience a magnet M50 (Biotronik Company) was placed over the device because repeated shocks had occurred. Placement of a magnet over the device failed to inhibit the delivery of S-ICD shocks. The Authors concluded that in patients with an S-ICD located deep in the subcutaneous tissue, it may be necessary to use several magnets to increase the probability of inhibiting shock delivery. In this experience, it is unclear whether the magnet was positioned appropriately, as providers did not appreciate an audible confirmation of the device deactivation. It is also possible that the magnet shifted during the prolonged CPR, leading to an undetected reactivation of shock therapy. The lack of appreciation of the device's audible tone, coupled with limited practitioner experience with this device, and the potential for antitachycardia therapies to be silently re-enabled should the magnet shift position, all represent potential mechanisms for these unexpected shocks $[31,32]$.

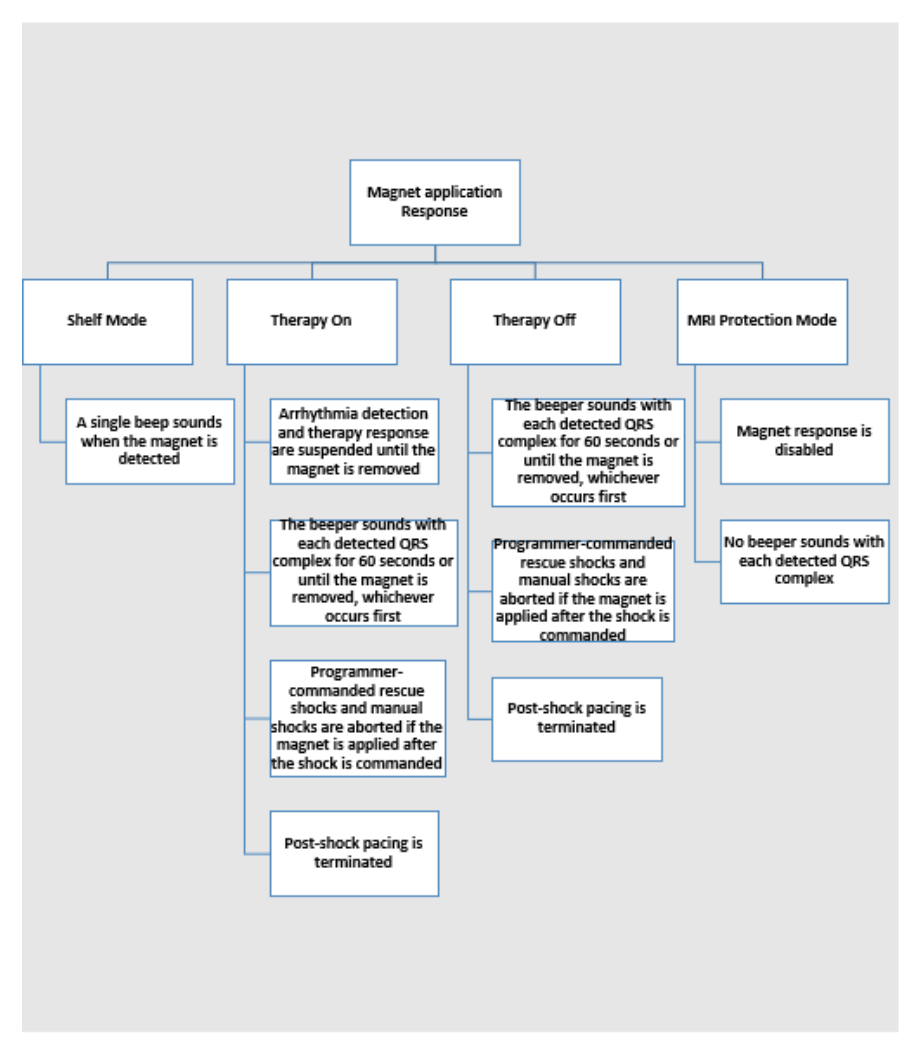

Figure 3: Flow-chart of S-ICD magnet application response. 
Our review provides information on the responses of S-ICD currently available to clinical magnets. Any clinical magnet model should be universal, making their use possible on all S-ICD models. Magnet use, while not fully reliable, has the advantage of being convenient, immediately reversible and expedites the patient's care [33-37]. The risk of human error and failure to re-enable shock therapies after the procedure is minimized. When a patient cannot provide a clearly accurate medical history or an identification card for the device, the position of the surgical pocket (subclavicular for the T-ICD and subaxillary for the S-ICD) can help to distinguish easily between the two devices. However, it is important to interrogate the device after the procedure to confirm that sensing and antitachycardia therapies are active.

\section{Key Teaching Points}

1. Patients with active S-ICD are increasingly admitted to Emergency Departments. Formal deactivation protocols, requiring appropriately trained staff and the presence of a magnet must be undertaken. Alignment with local cardiology services must also be in place to facilitate full device deactivation.

2. Magnet application for S-ICD it is usually located along the left mid axilla area. To ensure that you have the correct magnet placement location, ensure that you can feel the S-ICD under the skin. Place the magnet directly over the ICD and secure it with any type of tape. If the magnet were to lose connection with the S-ICD, the therapies you are suspending will no longer be suspended.

3. In Emergency Departments in case of inappropriate shocks and in the absence of a suitable EMBLEM S-ICD 3200 Programmer, it is recommended to use a specific 6860 or 4520 magnet to inhibit shock delivery. Any magnet must be placed above the upper or lower edge of the S-ICD device. Successful inhibition of shock delivery by the magnet is indicated acoustically with a beeping sound for 60 seconds.

4. It is recommended that S-ICD deactivation protocols are easily accessible and linked to their resuscitation policy. Heart Rhythm UK highlights the importance of a 24 hour cover for all aspects of S-ICD device management including device deactivation

\section{Conclusion}

This systematic review of literature demonstrates the feasibility of the application of any magnet model to inhibit the shock terapies of S-ICD devices. Even though there are conflicting data about the guidance in emergency, certain principles should be followed. Magnet application suppresses shock therapy only while in place and doesn't permanently alter S-ICD function. With the appropriate training, medical staff can help prevent further discomfort for these patients through the use of any model of magnet in a hospital and out of hospital setting. Emergency medical units should strongly consider the need to obtain and carry at least one clinical magnet model to control malfunctioning of S-ICD devices.

\section{Acknowledgement}

None.

\section{Conflict of Interest}

No conflict of interest.

\section{References}

1. Raul Weiss, Bradley P Knight, Michael R Gold, Angel R Leon, John M Herre, et al. (2013) Safety and efficacy of a totally subcutaneous implantablecardioverter defibrillator. Circulation 128: 944-953.

2. Louise RA Olde Nordkamp, Tom F Brouwer, Craig Barr, Dominic AMJ Theuns, Lucas VA Boersma, et al. (2015) Inappropriate shocks in the subcutaneous ICD: incidence, predictors and management. Int J Cardiol 195: 126-133.

3. Michael R Gold, Saleem Ahmad, Kevin Browne, Kellie Chase Berg, Lisa Thackeray, et al. (2012) Prospective comparison of discrimination algorithms to prevent inappropriate ICD therapy: primary results of the Rhythm ID Going Head to Head Trial. Heart Rhythm 9: 370-377.

4. Saini A, Ellenbogen KA (2017) Oversensing and inappropriate shock in a patient with subcutaneous implantable cardioverter defibrillator: What is the mechanism. Pacing Clin Electrophysiol 40: 897-899.

5. Dominic AMJ Theuns, Tom F Brouwer, Paul W Jones, Venugopal Allavatam, Steven Donnelley, et al. (2018) Prospective blinded evaluation of a novel sensing methodology designed to reduce inappropriate shocks by the subcutaneous implantable cardioverter-defibrillator. Heart Rhythm 15: $1515-1522$

6. Michael R Gold, Pier D Lambiase, Mikhael F El-Chami, Reinoud E Knops, Johan D Aasbo, et al. (2021) Primary Results from the Understanding Outcomes with the S-ICD in Primary Prevention Patients with Low Ejection Fraction (UNTOUCHED) Trial. Circulation 143: 7-17.

7. Alvarez-Acosta L, Romero-Garrido R, Hernandez-Afonzo J (2014) Inappropriate Defibrillator Shock in a Subcutaneous Device Secondary to Repetitive Muscle Contractions. Rev Esp Cardiol 67: 496-498.

8. Alessandro Corzani, Matteo Ziacchi, Mauro Biffi, Igor Diemberger, Cristian Martignani, et al. (2015) Inappropriate shock for myopotential over-sensing in patients with subcutaneous ICD. Indian Heart J 67: 5659.

9. Matthew M Zipse, William H Sauer, Paul D Varosy, Ryan G Aleong, Duy T Nguyen (2014) Inappropriate Shocks due to Subcutaneous Air in a Patient With a Subcutaneous Cardiac defibrillator. Circ Arrhythm Electrophysiol 7: 768-770.

10. Chieng D, Stewart B, Paul V (2018) Inappropriate shock from myopotentials due to subcutaneous defibrillator (S-ICD) movement conformed on fluoroscopy with subsequent device pocket revision. J Interv Card Electrophysiol 53: 263-265.

11. Asim S Ahmed, Parin J Patel, Shiv Bagga, Jasen L Gilge, Thomas Schleeter, et al. (2018) Troubleshooting electromagnetic interference in a patient with centrifugal flow left ventricular assist device and subcutaneous implantable cardioverter defibrillator. J Cardiovasc Electrophysiol 29: 477-481.

12. Yuji Ishida, Joshua E Payne, Michael E Field, Michael R Gold (2020) Electromagnetic interference from left ventricular assist devices in patients with subcutaneous implantable cardioverter-defibrillators. J Cardiovasc Electrophysiol 31: 1195-1201.

13.Jan-Hendrik van den Bruck, Arian Sultan, Tobias Plenge, Katharina Seuthe, Tim Mödder, et al. (2019) Incidence of myopotential induction 
in subcutaneous implantable cardioverter-defibrillator patients: is the oversensing issue really solved. Heart Rhythm 16: 1523-1530.

14. Kirsten M Kooiman, Reinoud E Knops, Louise Olde Nordkamp, Arthur A M Wilde, Joris R de Groot (2014) Inappropriate subcutaneous implantable cardioverter-defibrillator shocks due to T-wave oversensing can be prevented: implications for management. Heart Rhythm 11: 426-434.

15. Santomauro M, Petretta M, Riganti C ( 2020) Electrical Storm in Patients with Inappropriate Implantable Cardioverter-Defibrillator Therapy: Current Trends in Clinical Practice between Guidelines and Technology Progress. J Cardiol \& Cardiovasc Ther 15: 123-133.

16. Angelo Auricchio, J Harrison Hudnall, Edward J Schloss, Laurence D Sterns, Takashi Kurita, et al. (2017) Inappropriate shocks in singlechamber and subcutaneous implantable cardioverter-defibrillators: a systematic review and meta-analysis. Europace 19: 1973-1980.

17. Santomauro M, Petretta M, Riganti C (2020) Incidence of Inappropriate Subcutaneous Implantable Cardioverter Defibrillator disharges related to Electromagnetic Interferences. International Journal of Integrative Cardiology 2: 1-5

18. Colleen M McFaul, Stefan Lombaard, Vivek Arora, William C Van Cleve, G Alec Rooke, et al. (2020) Unexpected Shocks from subcutaneous implantable cardioverter-defibrillator despite attempted reprogramming and magnet use: a case report. A A Pract 14: e01178.

19. Sony Jacob, Sidakpal S Panaich, Rahul Maheshwari, John W Haddad, Benzy J Padanilam, et al. (2011) Clinical applications of magnets on cardiac rhythm management devices. Europace 13: 1222-1230.

20. Claire E Raphael, Michael Koa-Wing, Nolan Stain, Ian Wright, Darrel P Francis, et al. (2011) Implantable cardioverter-defibrillator recipient attitudes towards device deactivation: how much do patients want to know. Pacing Clin Electrophysiol 34: 1628-1633.

21. Porres JM, Laviñeta E, Reviejo C, Brugada J (2008) Application of a clinical magnet over implantable cardioverter defibrillators: Is it safe and useful. Pacing Clin Electrophysiol 31: 1641-1645.

22. Joshi GP (2009) Perioperative management of outpatients with implantable cardioverter defibrillators. Curr Opin Anaesthesiol 22: 701704.

23. Boston Scientific (2015) Using a magnet to temporarily inhibit S-ICD therapy manual.

24. Julia H Indik, J Rod Gimbel, Haruhiko Abe, Ricardo Alkmim-Teixeira, Ulrika Birgersdotter-Green, et al. (2017) 2017 HRS expert consensus statement on magnetic resonance imaging and radiation exposure in patients with cardiovascular implantable electronic devices. Heart Rhythm 14: e97-e153.

25. Can Hasdemir, Nayyar Shah, Arun P Rao, Helbert Acosta, Kagari Matsudaira, et al. (2002) Analysis of troponin I levels after spontaneous implantable cardioverter defibrillator shocks. J Cardiovasc Electrophysiol 13: $144-150$

26. Verena Semmler, Jürgen Biermann, Bernhard Haller, Clemens Jilek, Nikolaus Sarafoff, et al. (2015) ICD shock, not ventricular fibrillation, causes elevation of high sensitive troponin T after defibrillation threshold testing - the prospective, randomized, multicentre TropShock-Trial. PLoS One 10: e0131570.

27. Essandoh M, Daoud EG (2016) Perioperative Considerations for Patients With Subcutaneous Implantable Cardioverter Defibrillators Undergoing Noncardiac Surgery. J Cardiothorac Vasc Anesth 30: 756-761.

28. Berkowitz EJ,Pleimann BF, Rosenfels LE (2018) Subcutaneous implantable cardioverter defibrillator oversensing and shock delivery due to chest compression during CPR. Pacing Clin Electrophysiol 41: 1687-1690.

29. Peran D, Cmorej P, Pekara J (2019) Bystander hit by leakage current from S-ICD. Resuscitation 138: 297-298.

30. Patrik Cmorej, Eva Smrzova, David Peran, Tana Bulikova (2020) CPR Induced Inappropriate Shocks from a Subcutaneous Implantable Cardioverter Defibrillator during Out-of-Hospital Cardiac Arrest. Prehosp Emerg Care 24: 85-89.

31. Römers H, Van Dijk V, Balt J (2017) Erroneous magnet positioning leads to failure of inhibition of inappropriate shock during fast conducting atrial fibrillation episodes. Pacing Clin Electrophysiol 40: 741-743.

32. Schulman PM, Rozner MA (2013) Case report: use caution when applying magnets to pacemakers or defibrillators for surgery. Anesth Analg 117: 422-427.

33. Apfelbaum JL, Schulman PM, Mahajan A (2020) Practice advisory for the perioperative management of patients with cardiac implantable electronic devices: Pacemakers and implantable cardioverterdefibrillators: An updated report by the American Society of Anesthesiologists task force on perioperative management of patients with cardiac implantable electronic devices. Anesthesiology 114: 247261.

34. George H Crossley, Jeanne E Poole, Marc A Rozner, Samuel J Asirvatham, Alan Cheng, et al. (2011) The Heart Rhythm Society (HRS)/American Society of Anesthesiologists (ASA). expert consensus statement on the perioperative management of patients with implantable defibrillators, pacemakers and arrhythmia monitors: Facilities and Patient Management. Heart Rhythm 8: 1114-1152.

35. Yiliam F Rodriguez-Blanco, Fouad Souki, Evelyn Tamayo, Keith Candiotti (2013) Magnets and implantable cardioverter defibrillators: What's the problem. Ann Card Anaesth 16: 54-57.

36. Beinart R, Nazarian S (2013) Effects of External Electrical and Magnetic Fields on Pacemakers and Defibrillators: From Engineering Principles to Clinical Practice. Circulation 128: 2799-2809.

37. Janet Gifford, Karen Larimer, Celia Thomas, Patricia May, Stephen Stanhope, et al. (2014) Randomized controlled trials of perioperative ICD management: magnet application versus reprogramming. Pacing Clin Electrophysiol 37: 1219-1224. 